\title{
Molecular recognition in the gas phase
}

\author{
Marco Vincenti ${ }^{1}$, Ezio Pelizzetti1, Enrico Dalcanale ${ }^{2}$ and Paolo Soncini ${ }^{3}$ \\ ${ }^{1}$ Dipartimento di Chimica Analitica, Università di Torino, Via Giuria 5, \\ 10125 Torino, Italy. \\ 2Istituto di Chimica Organica, Università di Parma, Viale delle Scienze, \\ 43100 Parma, Italy. \\ 3Istituto Guido Donegani, Via Fauser 4, 28100 Novara, Italy.
}

\begin{abstract}
Several examples of molecular recognition taking place entirely in the gas-phase are demonstrated for the first time. Experiments involve highly selective host-guests complexations occuring between neutral counterparts inside the ion source of a mass spectrometer, where two cavitands are vaporized simultaneously in a gas-phase containing two candidate guests and an excess of methane. The relative gas-phase complexation constants are measured. The unique characteristic of this investigation method is that supramolecular interactions between specific functional groups of hosts and guests are made free from the solvation effects.
\end{abstract}

\section{INTRODUCTION}

Molecular recognition has been defined as a process involving both binding and selection of substrate by a given receptor molecule (ref. 1,2 ). When the substrate is a neutral organic molecule the design of the receptor requires, in addition to shape complementarity, the modulation of weak attractive forces. We focused our study on cavitands, which are synthetic macrocycles capable of molecular recognition (ref. 3). They form host-guest complexes with several substrates both in solution and in the solid state (ref. 4). However, solvent effects occurring in the condensed phase frequently hinder the formation and/or comprehension of supramolecular interactions. Recently, we demonstrated the occurrence of host-guest complexation also in the gas phase, in the absence of solvation effects (ref. 5).

In the present work we report the first examples of molecular recognition in the gas-phase. The usefulness of the innovative gas-phase approach to host-guest complexation is revealed by the high selectivity achieved, uncovering specific functional group interactions between hosts and guests. A systematic evaluation of the relative complexation constants $\left(\mathrm{K}_{\mathrm{a}}\right)$ in the gas-phase for various guests and hosts is also undertaken in this paper.

Cleft-shaped cavitand 1 and vessel-like cavitand 2 were selected for this study since their structural differences (two adjacent phenolic hydrogens on the rim of the cleft in 1 , a rigid concave surface in 2 ) are limited yet appropriate to enhance the specific role of each reactive site in the overall supramolecular interaction.

\section{EXPERIMENTAL SECTION}

Experiments were performed using a Finnigan-MAT 8400 double-focusing reverse geometry mass spectrometer, at an electron energy of $120 \mathrm{eV}$; the magnetic analyzer was scanned from 1100 to 1700 $\mathrm{Da}$ in $0.4 \mathrm{~s}$.. Some experiments were repeated with a Finnigan-MAT $95 \mathrm{Q}$ mass spectrometer, under similar conditions. Desorption chemical ionization mass spectrometry (DCI-MS) (ref. 6-8) was utilized to evaporate the cavitand, provide an atmosphere of guest molecules, and ionize the reaction products. 


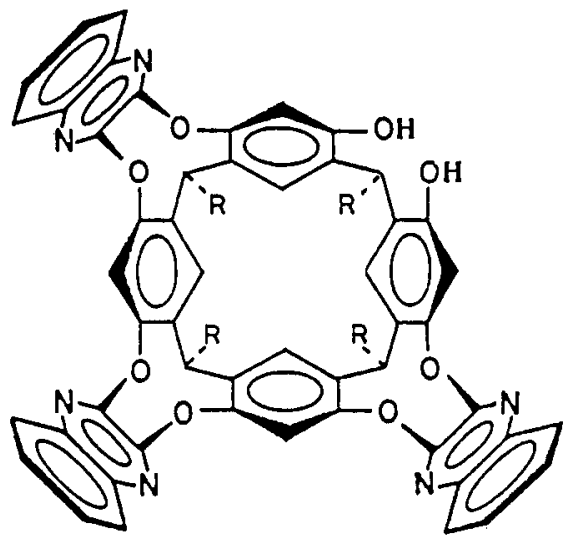

1

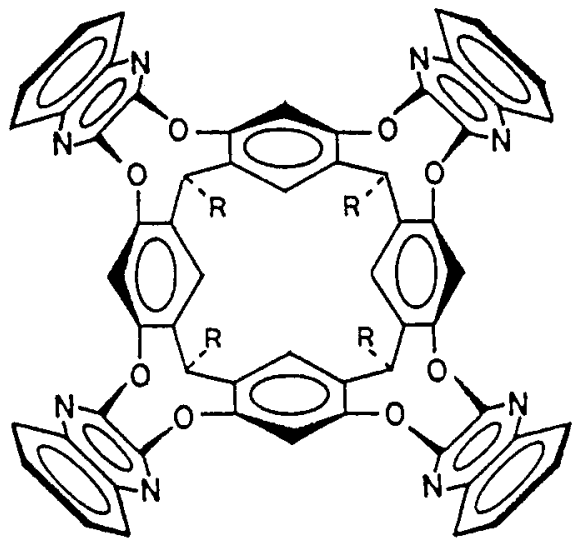

$\underline{2}$

The experimental procedure implies that few nanograms of a 1:1 mixture of the two hosts are placed on a rhenium wire and introduced into the mass spectrometer ion source (maintained at $80^{\circ} \mathrm{C}$ ) after evaporation of the solvent. In contrast with the previous study (ref. 5), where an atmosphere of pure guest was utilized, here the ion source is filled with about $0.5 \mathrm{mbar}$ of a gas mixture composed by methane ( $\cong 99 \%$ ) and small amounts of two guests ( $\cong 0.5 \%)$. This gas mixture is prepared by putting $1 \mathrm{ml}$ of a $1: 1$ guest mixture into a glass tube connected to the mass spectrometer ion source; air is pumped away after freezing the mixture in liquid nitrogen; upon equilibration at room temperature, the gas phase in the tube is analyzed by headspace gas chromatography (to determine the relative partial pressure of the candidate guests), using a flame ionization detector and the corresponding $G_{1}+G_{2}$ liquid mixture as calibrant. Then, methane is mixed in a pre-chamber, where relative pressures are adjusted by two regulating valves, while a third valve regulates the total pressure in the ion source. The percentage of methane, relatively to the guests, is estimated from the pressures read both on a Pirani gauge placed inside the ion source, and an ion gauge placed outside it. Once a stable pressure is reached inside the ion source, the two cavitands are simultaneously evaporated from the rhenium wire by fast heating induced by a rapidly increasing current $(40-80 \mathrm{~mA} / \mathrm{s})$. As soon as the cavitands are in the gas phase, they are thermally stabilized by multiple collisions with the bathing gas (ref. 9). Upon deexcitation, the cavitands undergo fast complexation with the two competing guests, both in large excess with respect to the host concentrations. The reaction products are revealed as negative ions upon capturing one electron thermalized by multiple collision with methane molecules.

\section{RESULTS AND DISCUSSION}

Figure 1 reports the mass spectrum arising from an experiment in which $\underline{1}$ and $\underline{2}$ are vaporized within an atmosphere of about $99 \%$ methane, $0.6 \%$ benzene and $0.4 \%$ n-butylamine. The molecular ions of the empty cavitands 1 and $\underline{2}$ are detectable respectively at $\mathrm{m} / \mathrm{z}=1202$ and $1328 \mathrm{Da}$, but they show a rather low abundance. Most part of both cavitands have interacted with the guests, giving rise to four major products, namely all the possible supramolecular complexes having benzene and n-butylamine as guests. It is noteworthy that 1 and 2 do not form complexes with methane despite its high concentration, while they extensively interact with benzene and n-butylamine, whose partial pressure within the reaction chamber is only $2-3 \times 10^{-3} \mathrm{mBar}$. Even more interesting is the fact that 1 forms complexes preferentially with n-butylamine, whereas 2 interacts much more extensively with benzene than with n-butylamine. This result can be explained by assuming that both the shape complementarity and the number of face-to-face and edge-to-face interactions favour the complexation of aromatics with 2 with respect to 1 (ref. 10,11 ). On the other hand, only 1 , bearing acidic phenolic hydrogens, should be capable of hydrogen bonding with the $-\mathrm{NH}_{2}$ group of $\mathrm{n}$-butylamine. 


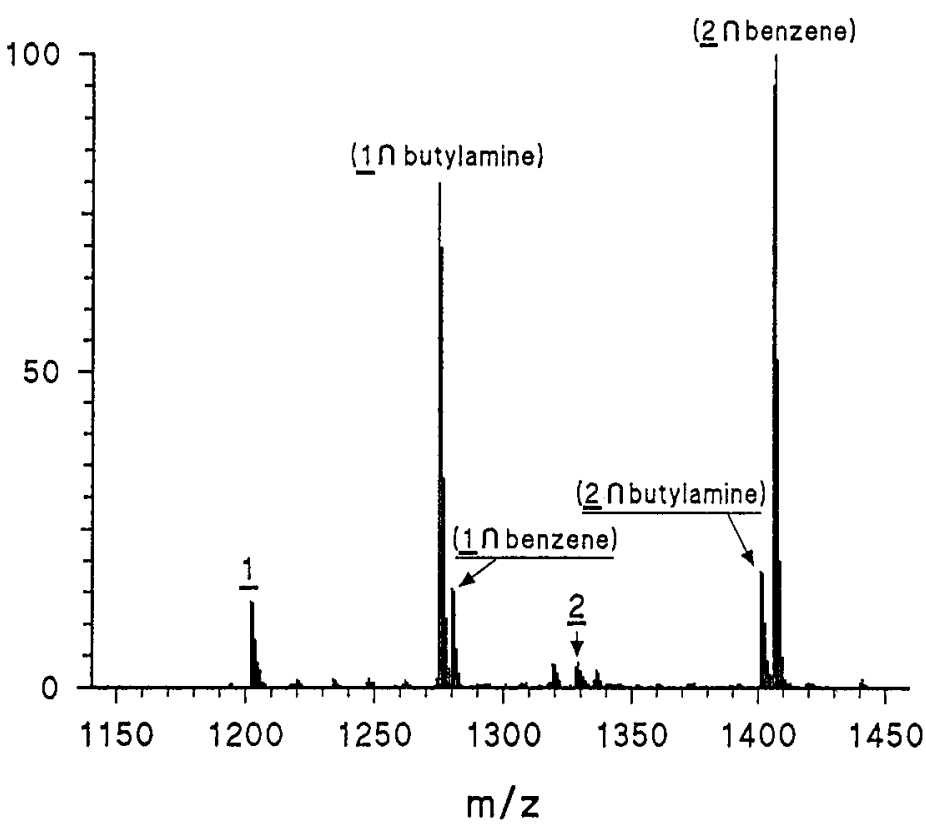

Fig. 1. Negative ion DCI mass spectrum of the products originating from the simultaneous evaporation of 1 and $\underline{2}$ in an atmosphere of methane $(99 \%)$, benzene $(0.6 \%)$ and n-butylamine $(0.4 \%)$.

The use of methane as the main gas-phase component provides extremely stable and reproducible conditions for the thermal deexcitation and ionization of the host and/or host-guest complex, which become independent of the specific guest chosen. Thus, the guests and their partial pressures can be freely varied without altering the ionization and deexcitation efficiencies, since an optimal CI pressure is maintained in the ion source. In a sense, methane can be considered as a "buffer gas".

A mixture of two guests is used in the experiments so that a competition for the receptors (the host molecules) is established, under controlled conditions. Therefore, it is made possible to evaluate the relative affinity of the two guests for a certain cavitand and to measure their relative complexation constant (see below). On the other hand, the two hosts do not compete with each other for the complexation of a certain guest, because the latter is present in large excess. However, the comparison between the $\mathrm{K}_{\mathrm{a}}$ for 1 and $\underline{2}$ is fundamental for understanding the nature of the host-guest interaction and to this purpose the overall conditions (temperature and pressures) should be identical for the two hosts. The best way to achieve this is to evaporate the cavitands together, so each is exposed to the same time period.

Two experimental evidences point to the thermodynamic control of the gaseous complexations occurring in the ion source of the mass spectrometer. If the ratio of the partial pressure of two guests is maintained constant while their absolute values are widely varied with respect to methane, the abundances for the two complexes change accordingly (with respect to the free host), but their ratio remains constant. Both a purely thermodynamic and a purely kinetic control of the reactions are consistent with these results, but the latter strongly contrasts with the relevant temperature effect observed for the complexation (ref. 5). When the chamber temperature is raised the extent of complexation decrease proportionally, pointing out that the reverse reaction is also a fast process. In conclusion, the system reaches the thermodynamic equilibrium before the products are ionized and analyzed or it is very close to it. 
The relative complexation constants can be calculated as follows:

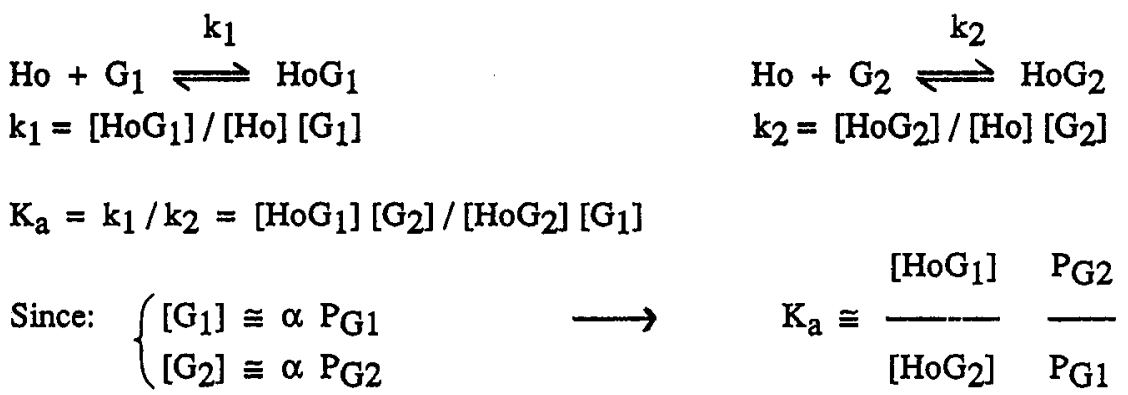

where $\mathrm{Ho}, \mathrm{G}_{1}, \mathrm{G}_{2}, \mathrm{HoG}_{1}, \mathrm{HoG}_{2}$ are the host, the two guests and the two complexes respectively, and $P_{G 1}, P_{G 2}$ are the partial pressures of the guests into the ion source. The ratio $\left[\mathrm{HoG}_{1}\right] /\left[\mathrm{HoG}_{2}\right]$ is measured by relative abundance of the corresponding peaks in the mass spectra. The ratio $P_{G 2} / P_{G 1}$ is determined by headspace gas chromatography, as mentioned above. Thus, the $\mathrm{K}_{\mathrm{a}}$ values for 22 triads $\left\{\mathrm{Ho}, \mathrm{G}_{1}, \mathrm{G}_{2}\right\}$ can be estimated, as listed in Table 1 .

Table 1. Relative complexation constants $\mathrm{K}_{\mathbf{a}}$, expressed as the ratio of the thermodynamic constants for the complexations between an host (Ho) and two different guests $\left(G_{1}, G_{2}\right)$. $K_{a}$ values are calculated from (1). All $\mathrm{K}_{\mathrm{a}}$ values have been averaged from 3-5 independent measurements of both $\left[\mathrm{HoG}_{1}\right] /\left[\mathrm{HoG}_{2}\right]$ and $\mathrm{P}_{\mathrm{G} 2} / \mathrm{P}_{\mathrm{G} 1}$, but in some experiments $\mathrm{K}_{\mathrm{a}}$ was determined at different $\mathrm{P}_{\mathrm{G} 2} / \mathrm{P}_{\mathrm{G} 1}$ ratios, by varying the liquid phase composition. When acetic acid was used as a candidate guest $\left(G_{2}\right)$, the mixed adduct $G_{1} \cap 1 \cap G_{2}$ was formed in significant abundance: the corresponding $\mathrm{K}_{\mathrm{a}}$ values are calculated from $\left(\left[\mathrm{HoG}_{1}\right]+\left[\mathrm{G}_{1} \mathrm{HoG}_{2}\right]\right) /\left(\left[\mathrm{HoG}_{2}\right]+\left[\mathrm{G}_{1} \mathrm{HoG}_{2}\right]\right)$ instead of $\left[\mathrm{HoG}_{1}\right] /\left[\mathrm{HoG}_{2}\right]$; values in parentheses refer to the usual counting.

\begin{tabular}{|c|c|c|c|}
\hline $\mathrm{HoG}_{1}$ & $\mathrm{~K}_{\mathrm{a}}$ & $\mathrm{HoG}_{1}$ & $\mathrm{~K}_{\mathrm{a}}$ \\
\hline (1nbenzene)-(1ncyclohexane) & 0.73 & (2nbenzene)-(2ncyclohexane) & 2.4 \\
\hline (1nbenzene)-(1ncyclohexene) & 0.53 & (2nbenzene)-(2ncyclohexene) & 1.0 \\
\hline (1 nbenzene)-(1 nacetonitrile) & 4.0 & 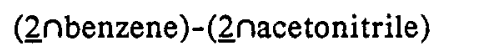 & 5.0 \\
\hline (1nbenzene)-(1nn-butylamine) & 0.13 & (므benzene)-( $\underline{2} \cap n$-butylamine) & 3.6 \\
\hline (1nbenzene)-(1 nethyl acetate) & 0.52 & 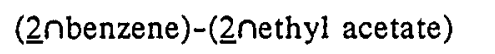 & $>50$ \\
\hline (1 nbenzene)-(1nmethyl acetate) & 20 & (2noenzene)-(2nmethyl acetate) & $>50$ \\
\hline (1ntoluene)-(1nn-butanol) & 0.015 & 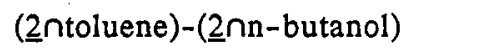 & 0.44 \\
\hline (1 nbenzene)-(1nethanol) & 4.1 & (2nbenzene)-(ㅁnethanol) & $>20$ \\
\hline$(1 \cap$ benzene $)-(1$ nacetic acid $)$ & $\begin{array}{c}0.08 \\
(<0.01)\end{array}$ & (2n nenzene)-(2nacetic acid) & 1.2 \\
\hline (1 ntoluene)-(1 nacetic acid) & $\begin{array}{l}0.35 \\
(0.02)\end{array}$ & (2ntoluene)-(2nacetic acid) & 1.5 \\
\hline (1ndioxane)-(1nacetic acid) & $\begin{array}{l}0.32 \\
(0.02)\end{array}$ & (2ndioxane)-(ㅁacetic acid) & 3.8 \\
\hline
\end{tabular}


The calculation of relative complexation constants is made on the basis of some reasonable assumption, that should, however, be mentioned: (i) the electron capture cross section of the complexes and the empty cavitands are approximately the same, i.e. the electron affinity of the host is not significantly modified by its interaction with the guest; (ii) rate constants for electron detachment reactions are also similar for cavitands and caviplexes; (iii) the residence time of ions into the ion source is 2-3 order of magnitude shorter than that of the neutral species, while rate constants for the complexation reactions are similar. These assumption are supported by the observation of similar experimental results in the positive and negative ion modes (ref. 5). A systematic study of the mechanistic aspects of supramolecular complexation in the gas-phase is in progress.

From Table 1, it can be deduced that cavitand 2 exhibits high affinity for aromatic (benzene, toluene) and unsaturated cyclic hydrocarbons (cyclohexene), which form complexes more extensively than cyclohexane, acetonitrile, butylamine, acetates and ethanol, presumably due to the establishing of strong $\pi-\pi$ interactions. Exceptions are n-butanol and acetic acid, also forming abundant adducts with 2. In these cases, some form of hydrogen bonding can be hypothesized.

In comparison with 2 , the behavior of 1 is more peculiar and intriguing. When 1 is mixed with a pure aromatic guest in the gas phase, only about half of the host molecules undergo complexation. A much more extensive complexation is observed when a guest capable of hydrogen bond is employed, such as acetic acid, n-butanol, n-butylamine and ethyl acetate. However, the occurrence of a strong hydrogen bond is far from explaining entirely the efficient complexation undergone by the compounds cited above, since other structurally similar molecules are unable to interact with 1 . The striking example of methyl- and ethyl acetates is presented in Fig. 2. While ethyl acetate forms complexes with 1 more extensively than benzene (Fig. 2-A), methyl acetate shows such a low affinity for 1 that the peak corresponding to their complex in Fig. 2-B is almost absent (m/z 1276). In contrast, the intensity of the ( 1 benzene) caviplex peak is enhanced by the virtual absence of a competitive guest. While the example in Fig. 1 gives prominence to the effects of structural differences in the hosts, Fig. 2 shows up the capability of the present gas-phase method to investigate phenomena of molecular recognition. In our interpretation, the carbonyl groups of methyl- and ethyl acetates form an hydrogen bond with the phenolic hydroxyls of 1 , but only in ethyl acetate the alcoholic residue is long enough that its terminal $-\mathrm{CH}_{3}$ group can interact with the quinoxaline opposite the phenolic hydroxyls. Thus, in this case complexation should be possible only when two distinct sites of supramolecular interaction are simultaneously present (ref. 12).

Polarizability effects should also be taken into account in supramolecular complexation even if, as far as hydrogen bonding is concemed, this effect is limited. For example, ethyl acetate is slightly more basic than methyl acetate in the gas-phase, but both of them have a much higher proton affinity than benzene (ref. 13).
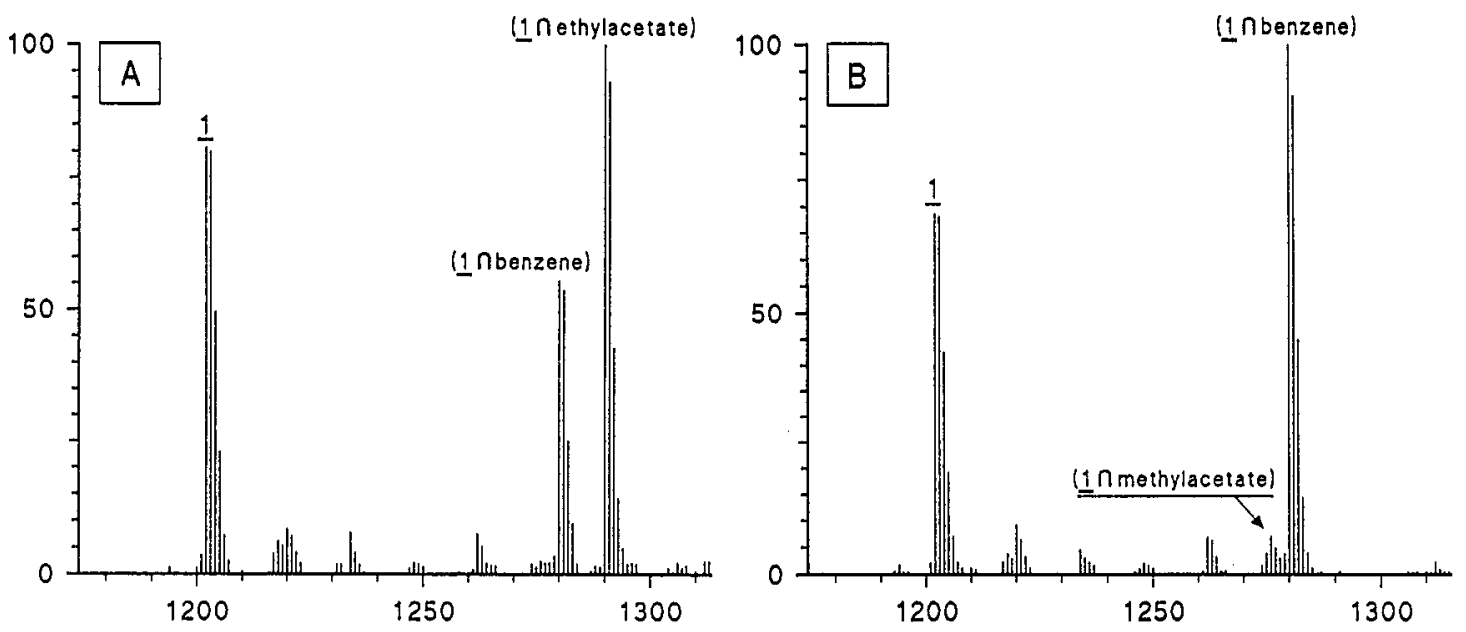

Fig. 2. Negative ion DCI mass spectra of the host-guest complexation products arising from 1 interacting with the following gaseous mixtures: (A) methane $99 \%$, benzene $0.52 \%$, ethyl acetate $0.48 \%$; (B) methane $99 \%$, benzene $0.4 \%$, methyl acetate $0.6 \%$. 
Further support to this interpretation is found in the experiments employing the mixtures toluenebutanol and benzene-ethanol as guests (Table 1). Benzene and toluene behave identically towards both 1 and 2 (ref. 5), and are indifferently used to match somewhat the partial pressure of the competing guest. Supramolecular interaction of n-butanol with 1 is extremely efficient: the corresponding complexation constant is more than 60 times higher than that with toluene, and only a few percent of 1 remains empty. Antithetically, negligible amount of complex between 1 and ethanol is formed, much less than with benzene. Thus, a rough estimation of the selectivity exhibited by 1 in the complexation of $n$-butanol versus ethanol exceeds 250 . Also in this case, the most likely explanation is that n-butanol is anchored to the host by a double interaction at the opposite extremes of its structure, namely an hydrogen bond between the hydroxylic functionalities of both molecules, and a $\mathrm{CH}_{3}-\pi$ interaction between the $\mathrm{CH}_{3}$ group of butanol and the quinoxaline ring of the host facing the - $\mathrm{OH}$. Ethanol is too short to undergo both these interactions and consequently its complex with 1 is much more labile. CPK model examination supports this interpretation.

Experiments employing acetic acid as guest are somehow peculiar. Although acetic acid gives rise to extremely stable adducts with 1 , due to the strong hydrogen bonds formed (in sharp contrast with 2 ), it is doubtful that its structure occupies the host cavity. In fact, a rather extensive formation of mixed complexes takes place, which include both acetic acid and the second guest (benzene, toluene, 1,4dioxane). A different behavior is displayed by 2 , which forms complexes in the usual $1: 1$ stoichiometry, both with acetic acid and the aromatic guest (or dioxane), whereas mixed complexes are produced only in low abundance. This observation can be explained assuming that in the latter case acetic acid have to be linked to the inside of the host cavity precluding further interactions with the second guest.

The whole set of experiments discussed so far demonstrate the presence of molecular recognition in the gas-phase. A new and promising tool for the investigation of host-guest interactions has been discovered, which is intrinsecally simple and presumably easy to rationalize.

\section{REFERENCES}

1. J.-M. Lehn, Angew. Chem. Int. Ed. Engl. 27, 89 (1988).

2. J. Jr. Rebek, Angew. Chem. Int. Ed. Engl. 29, 245 (1990).

3. D.J. Cram, J. Weiss, R. C. Hegelson, C. B. Knobler, A. E. Dorigo, K. N. Houk, J. Chem. Soc. Chem. Comm. 407 (1988).

4. E. Dalcanale, P. Soncini, G. Bacchilega, F. Ugozzoli, J. Chem. Soc. Chem. Comm. 500 (1989).

5. M. Vincenti, E. Dalcanale, P. Soncini, G. Guglielmetti, J. Am. Chem. Soc. 112, 445 (1990).

6. M. A. Baldwin, F.W. McLafferty, Org. Mass Spectrom. I, 1353 (1973).

7. R. J. Cotter, Anal. Chem. 52, 1589A (1980).

8. G. Guglielmetti, E. Dalcanale, S. Bonsignore, M. Vincenti, Rapid Commun. Mass Spectrom. 3, 106 (1989).

9. M. Vincenti, E. Pelizzetti, A. Guarini, S. Costanzi, Anal. Chem., in press.

10. A. V. Mühldorf, D. Van Engen, J. C. Warner, A. D. Hamilton, J. Am. Chem. Soc. 110, 6561 (1988).

11. A. D. Hamilton, N. Pant, A. V. Mühldorf, Pure Appl. Chem, 60, 533 (1988).

12. K. M. Neder, H. W. Whitlock Jr., J, Am. Chem. Soc. 112, 9412 (1990).

13. J. Hine, Structural Effects on Equilibria in Organic Chemistry, p. 150, Wiley, New York (1975). 